\title{
PIECEWISE LINEAR CRITICAL LEVELS AND COLLAPSING
}

\author{
BY
}

\author{
C. KEARTON AND W. B. R. LICKORISH
}

\begin{abstract}
In this paper the idea of collapsing, and the associated idea of handle cancellation, in a piecewise linear manifold are used to produce a version of Morse theory for piecewise linear embeddings. As an application of this it is shown that, if $n>2$, there exist triangulations of the $n$-ball that are not simplicially collapsible.
\end{abstract}

Working entirely within the P.L. category, we shall develop a proof of the fact that a locally unknotted embedding of a manifold $M$ in $Q \times I$, where $Q$ is a manifold with dimension not less than that of $M$, can be ambiently isotoped to become a critical level embedding. This is an embedding which, regarding $M$ as $M=$ handle + collar + handle + collar..., embeds each handle in a level of $Q \times I$ and each collar productwise along the $I$ direction. This is a folklore theorem which is fundamental to several important theories (e.g. [10], [4], [12]). As mentioned by Rourke [10], the result could probably be deduced from an amplification of the methods employed by Kuiper [8] and Kosiński [7], in proving that a P.L. function $f: M \rightarrow \mathbf{R}$ can be approximated by a function with only nondegenerate critical points (i.e., a P.L. analogue of Morse theory). That result can be deduced at once from the theory given here, by considering the graph of $f$ as an embedding of $M$ in $M \times I$. Our method of proof is essentially very simple, and uses only basic P.L. theory. We consider $M$ as a subcomplex of $Q \times I$, and a simplicial collapse of $Q \times I$ to $Q \times 0$; we drag a collar of $Q \times 0$ in $Q \times I$ up over the whole of $Q \times I$, following the collapsing sequence in the second derived subdivision of the triangulation, thus obtaining a new parametrisation of $Q \times I$. When the collar is dragged over a $p$-simplex of $M$, a level $p$-handle is introduced in this new parametrisation. Otherwise the proof consists of attention to detail. In Theorem 3, $M$ is replaced by $M \times I$, in order to consider concordances $M \times I \rightarrow Q \times I$. This theorem sets the stage for applying Rourke's proof of "concordance implies isotopy in codimension 3"', for the handles obtained for the critical level embedding of $M \times I$ actually cancel, as a handle decomposition of $M \times I$ on $M \times 0$, without any handle 'moves'. In conjunction with Rourke's proof, this theory also gives an alternative proof of unknotting spheres in codimension $\geq 3$.

Received by the editors May 14, 1971.

AMS 1970 subject classifications. Primary 57C35, 57C45; Secondary 57D70. 
The correlation of collapsing and critical level embeddings leads to a new result, namely that it is possible to triangulate the $n$-ball $(n \geq 3)$ by a nonsimplicially collapsible complex. This was proved by Bing [3] in the case when $n=3$; an erroneous proof of the general case appears in [5].

We shall be considering the type of handlebody theory that occurs most naturally in P.L. topology. In this theory, a handle decomposition of an m-dimensional manifold $M$ on an $m$-dimensional submanifold $M_{0}$ is a sequence of submanifolds $M_{0}, M_{1}, \cdots, M_{n-1}, M_{n}=M$, where $M_{i+1}$ is obtained from $M_{i}$ by adding a handle of some index $p=p(i)$. In symbols this is expressed as

$$
M_{i+1}=M_{i} \underset{a_{i}}{\cup}\left(B^{p} \times B^{m-p}\right)
$$

where $B^{r}$ is the $r$-ball, and $a_{i}: \partial B^{p} \times B^{m-p} \rightarrow \partial M_{i}$ is an embedding. This embedding is called the attaching map of the $i$ th handle $H_{i}^{p}$, and its image is the attaching tube. The $i$ th and $(i+1)$ th handles are said to cancel if they have indices $p$ and $p+1$ respectively, and

$$
\left(B^{p} \times 1\right) \times B^{m-p-1}=a_{i+1}^{-1}\left(B^{p} \times\left(B^{m-p-1} \times 0\right)\right)=a_{i+1}^{-1}\left(B^{p} \times \partial B^{m-p}\right),
$$

where $B^{p+1} \equiv B^{p} \times I$ and $B^{m-p} \equiv B^{m-p-1} \times I$.

There is, of course, an equivalent method of building up a manifold from handles, in which a copy of the product of the relevant boundary component and an interval is inserted before and after each handle addition. We shall regard the equivalence between these points of view in the following way. Suppose that $M$ is a manifold of dimension $(m-1)$ and that we add a handle $H^{p}$ to $M_{-} \times I$ with attaching map $a: \partial B^{p} \times B^{m-p} \rightarrow M_{-} \times 1$. Let $M$ be the manifold so formed and let $a\left(\partial B^{p} \times B^{m-p}\right)$ be $T \times 1$. Now $M=\left(M_{-} \times[0,1 / 2]\right) \cup\left(\left(M_{-}-\stackrel{\circ}{T}\right) \times[1 / 2,1]\right) \cup(T \times$ $[1 / 2,1]) \cup_{a}\left(B^{p} \times B^{m-p}\right)$. Let $\bar{H}^{p}$ be $\left(\Pi a\left(\partial \bar{B}^{p} \times 1 / 2 B^{m-p}\right) \times[1 / 2,1]\right) \cup_{a}\left(B^{p} \times 1 / 2 B^{m-p}\right)$ where $\Pi: M_{-} \times I \rightarrow M_{-}$is the projection; this is a $p$-handle added to $M_{-} \times[0$, 1/2]. But $(T \times[1 / 2,1]) \cup_{a}\left(B^{p} \times B^{m-p}\right)$ collapses to $(\partial T \times[1 / 2,1]) \cup(T \times 1 / 2) \cup \bar{H}^{p}$, so that, if $\partial_{-} \bar{H}^{p}$ and $\partial_{+} \bar{H}^{p}$ denote the attaching and belt tubes of the handle $\bar{H}^{p}$, the closure of $\left((T \times[1 / 2,1]) \cup_{a}\left(B^{p} \times B^{m-p}\right)\right)-\bar{H}^{p}$ is homeomorphic to $\left(\left((T \times 1 / 2)-\partial_{-} \bar{H}^{p}\right) \cup \partial_{+} \bar{H}^{p}\right) \times[1 / 2,1]$.

This homeomorphism can be explicitly recorded if desired, the homeomorphism being the identity on $\partial T \times[1 / 2,1]$. Thus $M=\left(M_{-} \times[0,1 / 2]\right) \cup \bar{H}^{p} \cup\left(M_{+} \times\right.$ $[1 / 2,1])$ where $M_{+}$is the manifold obtained from $M_{-}$by the surgery defined by $\bar{H}^{p}$. Thus we have $M$ in the form collar + handle + collar, and the product structures on the collars are determined by that of $M_{-} \times I$. Iterating this procedure, if we are given a handle decomposition of a manifold $M$ on $M_{-} \times I$, we have a canonical way of regarding $M$ as collar + handle $+\cdots+$ handle + collar. This will be called the associated collared handle decomposition. It is clear that if two of the original handles cancel then the associated handles cancel (in the usual sense) 
in the collared decomposition. Similarly, if $M$ is a closed manifold and we are given a handle decomposition of $M$ on the null set, an associated collared handle decomposition, 0 -handles + collar + handles $+\cdots+$ collar $+m$-handles, can be defined in the same way. If, in the above notation a pair of cancelling handles $H^{p}$ and $H^{p+1}$ were added to $M_{-} \times 1$, then $H^{p} \cup H^{p+1}$ becomes a ball attached to $M_{-} \times 1$ by a face. $M_{-} \times I$ is then homeomorphic to $\left(M_{-} \times I\right) \cup H^{p} \cup H^{p+1}$ by a homeomorphism which just 'stretches' a neighbourhood of the face in $M_{-} \times I$ over the ball. Hence if we wish, if a handle decomposition for $M$ has some consecutive pairs of cancelling handles, we can obtain a collared decomposition in which these cancelling pairs do not appear.

Definition. Let $M$ and $Q$ be manifolds, and let a handle decomposition of $M$ on $M_{-} \times I$ (or on $\phi$ ) be given. An embedding $e: M \rightarrow Q \times I$ is a critical level embedding for this decomposition if, with respect to the associated collared handle decomposition, e embeds the $i$ th handle in $Q \times t_{i}$ for some $t_{i} \in I$, and, on each collar, of the form $X \times I, e$ is the product of an embedding $X \rightarrow Q$ and a direction preserving embedding $I \rightarrow I$.

It is the aim of this paper to show that any embedding of $M$ in $Q \times I$ can be approximated by a critical level embedding. The following lemma describes a prototype situation.

Lemma 1. Let $\left(Q^{q-1}, M_{-}^{m-1}\right)$ be a manifold pair, $m<q$. Let the pair $(Q, M)$ be formed from $\left(Q_{-}, M_{-}\right) \times I$ be attaching a p-bandle pair $H^{p}=B^{p} \times\left(B^{q-p}, B^{m-p}\right)$ to $\left(\AA_{-}, \stackrel{\circ}{M}_{-}\right) \times 1$, where $\left(B^{q-p}, B^{m-p}\right)$ is a standard ball pair, and then adding a $(p+1)$-handle $H^{p+1}$ which cancels the p-bandle just added to $Q_{-} \times I$. Suppose that the attaching tube of $H^{p+1}$ does not meet $M$. Then there exists a bomeomorphism $b: Q_{-} \times I \rightarrow Q$ such that

(i) $b^{-1}: M \rightarrow Q_{-} \times I$ is a critical level embedding with respect to the bandle decomposition of $M$ on $M_{-} \times I$;

(ii) $b$ is the identity outside a regular neighbourbood of $\left(Q_{-} \times 1\right) \cap\left(H^{p} \cup\right.$ $\left.H^{p+1}\right)$.

Proof. As the handles added to $Q_{-} \times I$ cancel, we may write $H^{p}=B^{p} \times$ $\left(B^{q-p}, B^{m-p}\right)=B^{p} \times\left(B^{q-p-1} \times I, B^{m-p}\right)$ where $B^{m-p} \subset B^{q-p-1} \times 1 / 2$, and $H^{p+1}=$ $B^{p+1} \times B^{q-p-1}=\left(B^{p} \times I\right) \times B^{q-p-1}$. If $a_{1}$ and $a_{2}$ are the two attaching maps, we may assume that $a_{2}\left(\left(B^{p} \times 1\right) \times B^{q-p-1}\right)=B^{p} \times\left(B^{q-p-1} \times 0\right)$ but that otherwise the attaching tube of $H^{p+1}$ misses $H^{p}$. It may also be assumed that $a_{2}\left(\left(B^{p} \times 1\right) \times 1 / 2 B^{q-p-1}\right)=B^{p} \times\left(1 / 2 B^{q-p-1} \times 0\right)$. Now consider the following $p$ and $p+1$ handles added to $Q-\times[0,1 / 2]$ :

$$
\begin{aligned}
\bar{H}^{p} & =\left(B^{p} \times\left(1 / 2 B^{q-p-1} \times[0,1 / 2]\right)\right) \underset{a_{1}}{\cup}\left\{a_{1}\left(\partial B^{p} \times 1 / 2 B^{q-p-1} \times[0,1 / 2]\right) \times[1 / 2,1]\right\}, \\
\bar{H}^{p+1} & =\left(B^{p+1} \times 1 / 2 B^{q-p-1}\right) \underset{a_{2}}{\cup}\left\{a_{2}\left(\left(B^{p} \times 0 \cup \partial B^{p} \times I\right) \times 1 / 2 B^{q-p-1}\right) \times[1 / 2,1]\right\} .
\end{aligned}
$$


These form a cancelling pair of handles and $\bar{H}^{p}$ contains in its boundary the handle for $M$, in the associated collar handle decomposition of $M$. Thus $\bar{H}^{p} \cup \bar{H}^{p+1}$ is a $q$-ball $D$ with boundary the union of two $(q-1)$-balls $D_{-}$and $D_{+}$, where $D \cap Q_{-} \times 1 / 2=D_{-}$. Let $b: D_{-} \times[0,1 / 2] \rightarrow\left(D_{-} \times[0,1 / 2]\right) \cup D$ be a homeomorphism such that $h\left(D_{-}\right)=D_{+}$and $b$ is the identity near $\left(D_{-} \times 0\right)$ and on $\left(\partial D_{-} \times[0,1 / 2]\right)$. Let $E$ be the $q$-ball $H^{p} \cup H^{p+1}$ with the boundary $E_{-} \cup E_{+}$, where $E_{-}$and $E_{+}$are $(q-1)$-balls and $E \cap Q_{-} \times 1=E_{-}$. Now $E \cup\left(E_{-} \times[1 / 2,1]\right) \searrow\left(\partial E_{-} \times[1 / 2,1]\right) \cup$ $\left(E_{-} \times 1 / 2\right) \cup D \cup(M \cap E)$ and $M \cap E \searrow(M \cap D) \cup\left(M \cap\left(\left(E_{-} \times 1 / 2\right) \cup\left(\partial E_{-} \times[1 / 2,1]\right)\right)\right)$. Hence by standard results on regular neighbourhoods, the closure of $(E \cup(E+\times$ $[1 / 2,1]))-D$ is homeomorphic to $\left(\left(\left(E_{-} \times 1 / 2\right)-D_{-}\right) \cup D_{+}\right) \times[1 / 2,1]$ the homeomorphism being the identity on $\partial E_{-} \times[1 / 2,1]$ and compatible with the collar structure in the associated collar handle decomposition of $M$ (which was in fact obtained precisely in this way). Thus $b$ can be extended by the identity on $\left(\left(Q_{-}-D_{-}\right) \times[0,1 / 2]\right)$ $U\left(\left(Q_{-}-E_{-}\right) \times[1 / 2,1]\right)$ and by means of the above homeomorphism on $E_{-} \times[1 / 2,1]$. Thus $b: Q_{-} \times I \rightarrow Q$ has the required properties.

Addendum. If, in the statement of the lemma, $H^{p+1}$ were a handle pair $B^{p+1}$ $\times\left(B^{q-p-1}, B^{m-p-1}\right)$ which cancelled both the $p$-handle added to $Q_{-} \times I$ and that added to $M_{-} \times I$ then the situation becomes much simpler. For $H^{p} \cup H^{p+1}$ is then an unknotted ball pair attached to $Q_{-} \times 1$ by an unknotted face pair. Then a similar, but much less cumbersome argument (see e.g. Zeeman [15]) shows that $b$ can be constructed so that $b:\left(Q_{-}, M_{-}\right) \times I \rightarrow(Q, M)$ is a pairwise homeomorphism and property (ii) still holds. $b$ then simply 'stretches' the original collar pair over $(Q, M)$.

Suppose that $c$ is a collar of $Q \times 0$ in $Q \times I$, in other words, $c: Q \times I \rightarrow Q \times I$ is an embedding with $c \mid Q \times 0$ being the identity; $Q$ being now a closed manifold of dimension $(q-1)$. If we are given a cancelling handle decomposition for $Q \times I$ on $c(Q \times I)$ together with a procedure for cancelling these handles in pairs, then we can construct a homeomorphism $b: Q \times I \rightarrow Q \times I$, with $b \mid Q \times 0=1$, by 'stretching' the collar over each pair of cancelling handles (i.e. over a sequence of balls) in the usual way. We will say that an $b$ so constructed is associated with $c$ and the given handle cancelling procedure.

Lemma 2. If $h$ and $b^{\prime}$ are homeomorphisms of $Q \times I$ to itself both associated with a given collar $c$ of $Q \times 0$ and a given handle cancelling procedure, then $b$ and $b^{\prime}$ are isotopic keeping $Q \times 0$ fixed.

Proof. By induction on the number of pairs of cancelling handles it is sufficient to suppose that there is just one pair and that $b$ and $b^{\prime}$ are associated with collars $c$ and $c^{\prime}$ respectively, and with this single cancelling pair of handles, where $c(Q \times I)=c^{\prime}(Q \times I)$ and $c^{-1} c^{\prime}$ is isotopic to the identity keeping $Q \times 0$ 
fixed. Now $Q \times I=c(Q \times I) \cup B^{q}$ where $B^{q} \cap c(Q \times I)=B^{q-1}=\partial B^{q} \cap c(Q \times 1)$; $B^{q}$ is the union of the two handles. Let $\alpha, \alpha^{\prime}: c(Q \times I) \rightarrow Q \times I$ be homeomorphisms sending $B^{q-1}$ to $\partial B^{q}-B^{q-1}$ and which are the identity outside a regular neighbourhood of $B^{q-1}$. Then $\alpha^{-1} \alpha^{\prime}$ is isotopic to the identity, for the isotopy can be constructed first on the ball $B^{q-1}$ and then on the regular neighbourhood of $B^{q-1}$ in $c(Q \times I)$. But, $b=\alpha c$ and $b^{\prime}=\alpha^{\prime} c^{\prime}$ for some $\alpha$ and $\alpha^{\prime}$ with the above properties, and $c^{-1} \alpha^{-1} \alpha^{\prime} c^{\prime}$ is isotopic to the identity, hence $b$ and $b^{\prime}$ are isotopic.

If the manifold $Q \times I$ is triangulated as a combinatorial manifold in such a way that the projection $p: Q \times I \rightarrow Q \times 0$ is simplicial, it is well known that $Q \times I \searrow^{s} Q \times 0$ by a collapsing procedure that Zeeman [14] calls cylindrical. This procedure dictates that $A \times I \searrow^{s}(A \times 0) \cup(\partial A \times I)$ for all simplexes $A$ in $Q \times 0$ in some order of decreasing dimension. A second derived subdivision of the triangulation can be chosen so that the simplicial neighbourhood of $Q \times 0$ in the second derived is $Q \times[0, t]$ for some small $t$. Neighbourhoods in this second derived of vertices of the first derived give a handle decomposition of $Q \times I$ on $Q \times[0, t]$, the cylindrical collapsing procedure immediately induces a cancelling procedure for the handles. Define $c: Q \times I \rightarrow Q \times I$ to be the collar defined by taking $c$ to be the identity on the first factor and the linear map of $I$ onto $[0, t]$ on the second factor. We now have the necessary data for defining a homeomorphism $b: Q \times I \rightarrow Q \times I$ associated with $c$ and the handle cancelling procedure.

Lemma 3. If $b: Q \times I \rightarrow Q \times I$ is a homeomorphism associated with the data described above, then $b$ is isotopic to the identity keeping $Q \times 0$ fixed.

This result follows from the fact that $b$ can be chosen so that $b(A \times I)=A \times I$ for each simplex $A$ in $Q \times 0$, so that the isotopy can be constructed inductively over each $A \times I$. Furthermore, by Lemma 2 , it is immaterial which particular homeomorphism $b$ is constructed from the given data.

Although these results on homeomorphisms of $Q \times I$ to itself are basically straightforward, a certain amount of caution is advisable. It is known that in certain circumstances (see Siebenmann [11]) there exists a homeomorphism $\phi: Q \times I$ $\rightarrow Q \times I$, which is the identity on $Q \times 0$ and which is not isotopic to the identity homeomorphism. By the uniqueness of collars it can be assumed that $\phi$ is the identity on $Q \times[0, t]$ and, by standard results on subdivisions, including the theorem of J. H. C. Whitehead [13, Theorem 6], $Q \times I$ can be triangulated so that, for each simplex $A$ in $Q \times 0, A \times I \searrow^{s}(A \times 0) \cup(\partial A \times I)$, and $\phi\left(\phi^{-1} A \times I\right) \searrow^{s}$ $(A \times 0) \cup \phi\left(\phi^{-1}(\partial A) \times I\right)$. Hence we obtain two ways of simplicially collapsing $Q \times I$ to $Q \times 0$. This triangulation gives, via its second derived, a handle decomposition of $Q \times I$ on $c(Q \times I)$ where $c$ maps $Q \times I$ onto $Q \times[0, t]$ as before. The 
two collapsing procedures give two handle cancelling procedures of the same collection of handles. The first is associated (via $c$ ) with the identity homeomorphism, and the second with $\phi$.

Theorem 1. Suppose that $Q \times I$ is triangulated as a combinatorial q-manifold so that $Q \times I \searrow^{S} Q \times 0$. Let $M$ be a subcomplex, a closed combinatorial m-manifold, which is locally unknotted in the interior of $Q \times I$. Then there is a bomeophism $b: Q \times I \rightarrow Q \times I$ such that $b^{-1}: M \rightarrow Q \times I$ is a critical level embedding for a collared handle decomposition of $M$, associated with the second derived handle decomposition with some pairs of handles cancelled.

Proof. As already mentioned, the second derived of the triangulation of $Q \times I$ gives rise to the handle decomposition of $Q \times I$ on $Q \times[0, t]$, for some small $t$, and the collapsing implies that there is a cancelling procedure for these handles. Given $c: Q \times I \rightarrow Q \times[0, t]$, an associated $b: Q \times I \rightarrow Q \times I$ is constructed from the cancelling procedure as previously described. This $b$ is the composition of homeomorphisms each of which stretches a collar over a pair of cancelling handles $H^{p}$ and $H^{p+1}$, say. Now $H^{p}$ is the neighbourhood in the second derived subdivision of the barycentre of a $p$-simplex of $Q$. If this simplex belongs to $M$, then $H^{p}$ contains a $p$-handle of $M$ contained (by the local unknotting hypothesis) as a standard subhandle, as in the statement of Lemma 1. Similar considerations apply to $H^{p+1}$, which is the neighbourhood of the barycentre of a $(p+1)$-simplex of $Q$, which has the $p$-simplex as a face. If this $(p+1)$-simplex is in $M$ then $H^{p+1}$ contains a handle of $M$ which cancels the $p$-handle of $M$. Thus if $H^{p} \cup H^{p+1}$ meets $M$ we can apply either Lemma 1 in stretching the collar over $H^{p} \cup H^{p+1}$ or the addendum to Lemma 1 . It is then immediate that the resulting $b$ has the required properties.

As an application of Theorem 1 we prove in the following theorem that there exist noncollapsible triangulations of the $n$-ball.

Theorem 2. For any given integer $k$, there exists a triangulation of the $n$-ball, $n \geq 3$, sucb that the ktb derived subdivision of the triangulation is not simplicially collapsible.

Proof. It is well known that the group of the trefoil knot is the group with presentation $\{a, b: a b a=b a b\}$, and that, by spinning this knot [2], a knot of $S^{n-2}$ in $S^{n}$ is produced with the same group. Denote this knot by $A$, and let $m A$ denote the sum of $m$ copies of $A$.

It is shown, by Goodrick [5], that the $m$ th (Alexander) ideal of the group of $m A$ is not the whole of the group ring. Thus this group has no presentation with fewer than $m+1$ generators.

Now consider the knot $m A$ as a polyhedron $S^{n-2}$ contained in the interior 
of a ball $B^{n}$ (rather than $S^{n}$ ), where $m$ is so large that it exceeds the number of vertices in the $k$ th derived of the boundary of an $(n-1)$-simplex. By Armstrong [1], $B^{n}$ can be triangulated so that $S^{n-2}$ is a subcomplex, this subcomplex being isomorphic to the boundary of an $(n-1)$-simplex. Suppose the $k$ th derived of $B^{n}$ is simplicially collapsible to a point $P$. It may be assumed, without loss of generality, that $P \in B^{n}-S^{n-2}$. Excising a very small neighbourhood $N$ of $P$ and using Theorem 1, there is a homeomorphism $b: S^{n-1} \times I \rightarrow B^{n}-\stackrel{\circ}{N}$ such that $b^{-1}$ : $S^{n-2} \rightarrow S^{n-1} \times I$ is a critical level embedding for a handle decomposition associated with the $k$ th derived of the triangulation of $S^{n-2}$. This has fewer than $m 0$ handles. Now an immediate generalisation of the methods used by Fox [4] for calculating the group of a knot of $S^{2}$ in $S^{4}$ shows that the fundamental group of $\left(s^{n-1} \times I\right)-b^{-1} S^{n-2}$ has a presentation with one generator for each 0 -handle of the critical level embedding, and one relation for each 1 -handle. Therefore there is a presentation of the group of $m A$ with fewer than $m$ generators, which is a contradiction. Hence the triangulation of $B^{n}$ was not simplicially collapsible.

Theorem 3. Let $M^{m}$ and $Q^{q}$ be closed P.L. manifolds, $m<q$, let $e: M \times I \rightarrow$ $Q \times I$ be a concordance of $M$ in $Q$ (i.e. a locally unknotted embedding such that $e^{-1}(Q \times t)=M \times t$ for $t=0$ or 1$)$. Then there exists a bomeomorphism $b: Q \times I \rightarrow$ $Q \times I$ such that $b$ is is otopic to the identity rel $Q \times 0$, and $b^{-1} e$ is a critical level embedding for some cancelling decomposition of $M$ on a collar of $M \times 0$.

Proof. The proof of this theorem is very similar to that of Theorem 1. Firstly, by results on compatible collars [6, Chapter VI] it may be assumed that $e(x, u)=\left(e_{0} x, u\right)$ for all $x \in M$ and all $u \leq t_{0}$ for some small $t_{0}$, where $e_{0}: M \rightarrow Q$ is defined by $\left(e_{0}(x), 0\right)=e(x, 0)$. It may also be assumed, after a small move of $e$ into general position, that the projection $Q \times\left[t_{0}, 1\right] \rightarrow Q \times t_{0}$ is nondegenerate on $e\left(M \times\left[t_{0}, 1\right]\right)$. Triangulate $M \times I$ and $Q \times I$ so that $e$ is simplicial, $Q \times\left[0, t_{0}\right]$ is a subcomplex of $Q \times I$, and $M \times I \searrow^{s} M \times\left[0, t_{0}\right]$. Subdivide so that the projection $Q \times I \rightarrow Q \times 0$ is simplicial, and take the subdivision induced by $e$ of $M \times I$; these are the triangulations we need. There is then a cylindrical collapse $Q \times I$ $\searrow^{s} Q \times 0$, so starting with a collar $c: Q \times I \rightarrow Q \times[0, t], t<t_{0}$, we can form a homeomorphism $b: Q \times I \rightarrow Q \times I$ associated with $c$ and the cancelling procedure for the handles of the second derived subdivision of $Q \times I$, defined by the collapse. This $b$ is isotopic to the identity, by Lemma 3, rel $Q \times 0$. Exactly as in the proof of Theorem $1, b$ can be chosen so that $b^{-1} e$ is in critical level position with respect to the handles of the second derived subdivision of $M \times 1$, with some pairs of handles cancelled. However, the only handles that are cancelled are those corresponding to the barycentres of a $p$-simplex and a $(p+1)$-simplex, such that the images under $e$ of these simplexes are collapsed away together in the 
collapsing procedure. But the collapse is cylindrical and $Q \times\left[t_{0}, 1\right] \rightarrow Q \times t_{0}$ is nondegenerate on $e\left(M \times\left[t_{0}, 1\right]\right)$, so such a pair of simplexes occur only when they both belong to $M \times\left[0, t_{0}\right]$. Hence, $b^{-1} e$ is in critical level position with respect to the handle decomposition of $M \times I$ on the second derived neighbourhood of $M \times$ $\left[0, t_{0}\right]$.

Now, the triangulation of $M \times I$ is a subdivision of one which collapses simplicially to $M \times\left[0, t_{0}\right]$, and a further subdivision of $M \times I$ would restore this property. Hence by subdividing the handles of the given decomposition of $M \times I$ on $M \times\left[0, t_{0}\right]$, we have a cancelling decomposition. (Subdivision of a handle involves replacing the handle by a handle decomposition of it relative to its attaching tube.) But if we have a handle embedded in a level of $Q \times I$, it is easy to adjust this embedding so that, after an isotopy, the handles of a subdivision of that handle are embedded in critical level fashion. Hence the result is proved. In fact, this last manoeuvre can be avoided by a dexterous use of the subdivision theorem of J. H. C. Whitehead [13, Theorem 6]. For we may subdivide the triangulation of $Q \times I$ used above so that $M \times I \searrow^{s} M \times\left[0, t_{0}\right]$, $e$ is simplicial, and $Q \times I \searrow^{s} Q \times 0$, the latter collapse being cylindrical with respect to some P.L. cell structure on $Q$. The handle decomposition of $M \times I$ must now be one which cancels without any handle moves. Note that the way in which a simplex of $M \times I$ appears in the first collapsing sequence is unrelated to the way it appears in the second, so no information is obtainable, from this method, concerning the possibility of having a pair of cancelling handles of $M \times I$ in adjacent levels of $Q \times I$.

Theorem 3 says that the embedding $e$ can be ambiently isotoped to become a critical level embedding. It is natural to ask whether, given an $\epsilon>0$, this isotopy can be constructed so as to be an $\epsilon$-isotopy. The isotopies used in the proof of the theorem are all small, with the possible exception of the isotopy of $b$ to the identity given by, Lemmas 2 and 3. In the proof of Lemma 3, if $A$ were a simplex of $Q \times 0, A \times I$ always remained in itself during the isotopy. In the proof of Lemma 2, assuming that it is referring to a handle cancelling procedure associated with a cylindrical collapsing, $A \times I$ is never isotoped outside (star $A$ ) $\times I$. Thus it can be ensured, by taking a fine enough triangulation of $Q \times I$ in the first instance, that no point is moved more than $\epsilon$ by the isotopies in the 'direction' of the $Q$ component. We have lost control of distances in the $I$ direction. However, the whole theorem clearly works for any cobordism in place of $M \times I$, so we can divide $Q \times I$ into a number of consecutive slabs of the form $Q \times[\alpha, \beta]$, where $\beta-\alpha<\epsilon$, so that $e(M \times I)$ meets each slab in a cobordism and apply the theorem to each slab in turn. The result is a true $\epsilon$-version of the theorem, i.e. $b$ is $\epsilon$ ambient isotopic to the identity.

Now let $e: M \times I \rightarrow Q \times I$ be a locally unknotted concordance between 
embeddings $e_{0}$ and $e_{1}: M \rightarrow Q$ and suppose $q-m \geq 3$. We can isotope $e$ to a critical level embedding with respect to a cancelling handle decomposition and then apply Rourke's technique immediately and use the cancelling of the se handles to show that $e_{0}$ is ambient isotopic to $e_{1}$. In this method, for a pair of cancelling handles of $M \times I$, an isotopy of $e$ is constructed to send $e$ to a critical level embedding for the collared handle structure corresponding to the structure with the given pair cancelled. Repeating this for each pair of handles results in a final embedding $e^{\prime}$, ambient isotopic to $e$, with $e^{\prime}(M \times I)=\left(e_{0} \times \mathrm{id}\right)(M \times I)$ and $e^{\prime} \mid M \times 0=\left(e_{0} \times\right.$ id $) \mid M \times 0$. However, the handle cancelling procedure for $M \times I$ on the collar of $M \times 0$ in $M \times I$ produced in Theorem 3 can be taken to be associated with a subdivision of a cylindrical collapse. Application of Lemmas 2 and 3 shows that $\left(e^{\prime}\right)^{-1}\left(e_{0} \times \mathrm{id}\right): M \times I \rightarrow M \times I$ is isotopic to the identity, and thus by an isotopy covering theorem, $e^{\prime}$ is ambient isotopic to $e_{0} \times$ id. If now $e$ is within $\epsilon$ of the imbedding $e_{0} \times \mathrm{id}: M \times I \rightarrow Q \times I$, then we may assume that the critical level embedding is also within $\epsilon$ of $e_{0} \times$ id. It is expected that, when the details of Rourke's proof are published, they will apply in this situation to show that $e_{0}$ and $e_{1}$ are ambient $\epsilon$-isotopic. In that case we should have an alternative proof of Miller's result [9] that in codimension three or more, given a proper embedding of a ball in a ball and an $\epsilon>0$, there exists a $\delta$ such that any other proper embedding within a $\delta$ of the first is ambient $\epsilon$-isotopic to the first embedding (the relevant $\epsilon$-concordance being constructed conewise).

\section{REFERENCES}

1. M. A. Armstrong, Extending triangulations, Proc. Amer. Math. Soc. 18 (1967), 701-704. MR $36 \# 4565$.

2. E. Artin, Zur Isotopie zweidimensionalen Flächen im $R_{4}$, Abh. Math. Sem. Univ. Hamburg 4 (1926), 174-177.

3. R. H. Bing, Some aspects of the topology of 3-manifolds related to the Poincaré conjecture, Lectures on Modern Math., vol. 2, Wiley, New York, 1964, pp. 93-128. MR $30 \# 2474$.

4. R. H. Fox, A quick trip through knot theory, Proc. Topology of 3-Manifolds and Related Topics (Univ. of Georgia Inst., 1961), Prentice-Hall, Englewood Cliffs, N. J., 1962, pp. 120-167. MR 25 \#3522.

5. R. E. Goodrick, Non-simplicially collapsible triangulations of $I^{n}$, Proc. Cambridge Philos. Soc. 64 (1968), 31-36. MR 36 \#3338. $40 \# 2094$.

6. J. F. P. Hudson, Piecewise linear topology, Benjamin, New York, 1969. MR

7. A. Kosiński, Singularities of piecewise linear mappings. I. Mappings into the real line, Bull. Amer. Math. Soc. 68 (1962), 110-114. MR 24 \#A2389.

8. N. H. Kuiper, Non-degenerate piecewise linear functions, Rev. Roumaine Math. Pures Appl. 13 (1968), 993-1000. MR 38 \#2789.

9. R. T. Miller, Close isotopies on piecewise-linear manifolds, Trans. Amer. Math. Soc. 151 (1970), 597-628. MR 42 \#1127.

10. C. P. Rourke, Embedded handle theory, concordance and isotopy, University of Warwick, 1968 (preprint).

11. L. C. Siebenmann, Torsion invariants for pseudo-isotopies on closed manifolds, 
Notices Amer. Math. Soc. 14 (1967), 942-943. Abstract \#67T-674.

12. A. G. Tristram, Slice links, Cambridge Ph. D. Thesis, 1966.

13. J. H. C. Whitehead, Simplicial spaces, nuclei and m-groups, Proc. London Math. Soc. 45 (1939), 243-327.

14. E. C. Zeeman, Seminar on combinatorial topology 1963-1965, Inst. Hautes Études Sci., Paris.

15._-_-, Unknotting combinatorial balls, Ann of Math. (2) 78 (1963), 501-526. MR $28 \# 3432$.

DEPARTMENT OF PURE MATHEMATICS, UNIVERSITY OF CAMBRIDGE, CAMBRIDGE, ENGLAND

Current address (C. Kearton): Department of Mathematics, Princeton University, Princeton, New Jersey 08540 